\title{
Leishmania infantum and Dirofilaria immitis coinfection in dogs in Greece
}

\author{
PANTELIS NTAIS ${ }^{1}$, VASILIKI CHRISTODOULOU ${ }^{1,2}$, EMMANOUIL DOKIANAKIS ${ }^{1}$ \\ and MARIA ANTONIOU ${ }^{1} *$ \\ ${ }^{1}$ Laboratory of Clinical Bacteriology, Parasitology, Zoonoses and Geographical Medicine, Faculty of Medicine, University \\ of Crete, Crete, Greece \\ ${ }^{2}$ Veterinary Services of Cyprus, Nicosia, Cyprus
}

(Received 1 September 2016; accepted 7 October 2016)

\begin{abstract}
SUMMARY
Leishmaniasis and dirofilariasis are parasitic diseases of humans and dogs, worldwide, and they are often found as coinfections in endemic areas. Cases of human and canine dirofilariasis have being reported in Greece and leishmaniasis is endemic in most prefectures in humans and dogs. In most cases, dirofilariasis is established by parasitological (the modified Knott's test) and/or immunological methods, whilst for leishmaniasis molecular techniques and culture are also used. During an epidemiological study in Greece, $22 \cdot 1 \%$ of the 5772 dogs studied were found positive by serology for Leishmania. Blood cultures of 165 (12.94\%) of these animals produced Leishmania promastigotes and $26(2 \cdot 03 \%)$ Dirofilaria microfilariae (L1), whilst only in two $(0 \cdot 16 \%)$ both Leishmania and Dirofilaria L1 appeared. The aim was to assess coinfections by the two parasites in dogs in Greece, the isolation and survival of Dirofilaria microfilariae and Leishmania promastigotes using clotted blood (a fast, simple and low-cost method) and the survival potential of the two parasites in coexistence, in vitro.
\end{abstract}

Key words: isolation, dog blood, Dirofilaria microfilariae, Leishmania infantum, coinfection.

\section{INTRODUCTION}

Leishmaniasis and dirofilariasis are both vectorborne diseases. Leishmania (Trypanosomatida, Trypanosomatidae) uses sand flies (Phlebotomus spp.) (WHO, 1997) and Dirofilaria (Spirurida, Onchocercidae) uses mosquitoes (species of Aedes, Culex, Anopheles and Mansonia) for their transmissions to mammalian hosts (Otto and Jachowski, 1981). During a blood meal, an infected sand fly introduces promastigotes to the warm-blooded host, whilst the infected mosquito introduces third-stage filarial larvae (L3) of Dirofilaria. In the mammalian host, promastigotes enter macrophages and inside the cell they replicate as amastigotes, whilst the L3 larvae undergo two moults to become adults who reside in pulmonary arteries and the heart. For 5-10 years the female worms produce microfilariae (L1), which enter into the peripheral blood (McCall et al. 2008). Infected macrophages and free L1 are then taken up by their vectors to complete their life cycles. In areas where hosts, infected by these parasites, and competent insect vectors exist, coinfections may arise (Aresu et al. 2007; Maia et al. 2015). It appears that changes in climate and human activities aid in the geographical spread of these zoonoses and human,

* Corresponding author: Laboratory of Clinical Bacteriology, Parasitology, Zoonoses and Geographical Medicine, Faculty of Medicine, University of Crete, Crete, Greec. E-mail: antoniou@med.uoc.gr as well as $\operatorname{dog}$ and feline; cases are increasing in numbers even in areas where the problems were not reported before (Orihel and Eberhard, 1998; Pampiglione and Rivasi, 2000; Dujardin, 2006; Maroli et al. 2008; Tanczos et al. 2012).

Five species of filarial nematodes: Dirofilaria immitis, Dirofilaria repens, Dipetalonema reconditum, Dipetalonema grassii and Acanthocheilonema reconditum have being recovered from dogs (Genchi et al. 2011); and Leishmania infantum (synonym: Leishmania chagasi in the New World) is the most common Leishmania species causing canine leishmaniasis (CanL), worldwide (Alvar et al. 2012). In Greece, D. immitis has been reported in dogs (Polizopoulou et al. 2000; Sinanis et al. 2012) and D. repens (D. conjunctivae) in humans (Vakalis and Himonas, 1997), whilst L. infantum comprises one of the most important parasitic diseases in dogs in most parts of Greece, and an important zoonosis for public health (Antoniou et al. 2009; Christodoulou et al. 2012; Ntais et al. 2013).

The aim of this work was to evaluate the geographical distribution of coinfected dogs in Greece, to isolate the two parasites from the blood of dogs, using a simple method, and to assess their survival and interaction in vitro.

\section{MATERIALS AND METHODS}

\section{Study area and animal sampling}

The study was conducted in all (54) prefectures of Greece where 63 veterinarians, collaborating with

Parasitology Open (2016), Vol. 2, e17; page 1 of 5. (C) Cambridge University Press 2016. This is an Open Access article, distributed under the terms of the Creative Commons Attribution licence (http://creativecommons.org/licenses/by/4.0/), which permits unrestricted re-use, distribution, and reproduction in any medium, provided the original work is properly cited. 
our laboratory, provided dog samples from 5772 animals visiting their clinic for any reason: vaccination, haircut, nail cut, deworming, general check-up, treatments and other purposes, without discrimination (Ntais et al. 2013). The animals were examined clinically and biological material was collected, after the written consent of the owner, and a questionnaire with personal, epidemiological and clinical data for each dog was completed. Biological samples, including peripheral blood without anticoagulants from all dogs taking part in the study, were collected and stored in sterile tubes at $4{ }^{\circ} \mathrm{C}$ until transfer to the laboratory for processing. The maximum number of samples reached 300 per prefecture and depended on the size of the prefecture and dog population (Ntais et al. 2013).

\section{Serology}

Dog sera were tested serologically using anti-dog, anti-immunoglobulin $\mathrm{G}$ antibodies by an indirect immunofluorescent antibody test (IFAT, Leishmania SPOT IF, BioMerieux, France). A series of 2 -fold serum dilutions, starting from $1 / 40$ were performed and a cut-off titre of $\geq 1 / 160$ was regarded positive for dog sera since most animals lived in endemic areas (Ferroglio et al. 2002).

\section{Polymerase chain reaction (PCR) and PCR- restriction fragment length polymorphism (PCR-RFLP)}

The PCR for the detection of Leishmania DNA was carried out on whole blood, lymph node and/or spleen tissue from dogs, according to availability. The QIAamp DNA Blood Mini kit (QIAGEN, Hilden, Germany) and DNeasy Tissue kit (QIAGEN) were used for DNA extraction from blood and tissue, respectively. Primers T2 and B4 were used as described previously with few modifications (Minodier et al. 1997; Christodoulou et al. 2012). The Leishmania ITS1 region followed by a HaeIII restriction endonuclease digestion of the positive PCR products (Schönian et al. 2003) was amplified in order to identify the parasite species infecting the dogs. All samples were tested in duplicates. Positive controls were used in all PCR assays, which consisted of DNA extracted from L. infantum (MCAN/GR/2009/GD70) and Leishmania tropica (MCAN/GR/2009/GD52), the two Leishmania spp. found in Greece, which had derived from Greek dogs and humans, respectively, and typed by enzyme electrophoresis (Ntais et al. 2013; Karayiannis et al. 2015). Negative controls included samples from healthy dogs born and lived in nonendemic areas in Crete (at $>1000 \mathrm{~m}$ altitude), that had being tested for Leishmania serologically, by PCR and culture and proved negative.

\section{Parasite culture}

Parasite culture was performed using blood, without anticoagulants, of 1275 animals, which were positive both by IFAT and PCR. Sterile clotted blood $\left(0.5 \mathrm{~cm}^{3}\right.$ obtained from the core of the sample) was suspended in $3 \mathrm{~mL}$ RPMI 1640 culture medium containing $25 \mathrm{~mm}$ Hepes buffer, supplemented with $2 \mathrm{~mm}$ glutamine (GIBCO Invitrogen, Grand Island, NY), $10 \%$ heat inactivated fetal bovine serum (FBS - GIBCO Invitrogen), $100 \mathrm{IU} \mathrm{mL}^{-1}$ penicillin, $100 \mathrm{mg} \mathrm{mL}^{-1}$ streptomycin (Roche Diagnostics, Indianapolis, IN), and 5\% filtered human urine, and incubated, in culture flasks, at $26^{\circ} \mathrm{C}\left( \pm 1^{\circ} \mathrm{C}\right)$ (Howard et al. 1991; WHO, 1991).

\section{Parasite identification}

Leishmania was identified using PCR-RFLP, as described above, and Dirofilaria was identified after microscopic examination of the microfilariae using morphometric characters (body size and shape) (Magnis et al. 2013).

\section{RESULTS}

Of the 5772 randomly selected dogs examined for leishmaniasis by IFAT, and PCR, 1275, (22.09\%) were positive both by serology and PCR (Ntais et al. 2013). PCR-RFLP showed the protozoan parasite to be L. infantum and morphometric characters of the microfilariae (length, 290-332 $\mu \mathrm{m}$; width, 6-7 $\mu \mathrm{m}$; straight body and tail; tapered anterior end) showed the Nematode parasite to be D. immitis (Magnis et al. 2013).

Culture, performed using clotted blood of only the 1275 PCR and IFAT-positive animals, resulted in 165 positive for Leishmania (12.94\%) and 26 positive for Dirofilaria $(2 \cdot 04 \%)$ culture media. In two of these cultures (both from the island of Corfu, with animal IFAT titres against Leishmania 1/640 and $1 / 5120)$, both Leishmania and Dirofilaria emerged $(0 \cdot 16 \%)$.

The 26 dogs, from the blood of which Dirofilaria L1 was isolated, were 16 males and 10 females; none was a stray animal. Their age was: $6>3$ years, 12 between 4 and 6 years, 4 between 7 and 9 years, and $4>10$ years. Their weight was: $4>10 \mathrm{~kg}, 10$ between 11 and $20 \mathrm{~kg}, 10$ between 21 and $30 \mathrm{~kg}$, and $2>30 \mathrm{~kg}$. All 26 dogs presented antibodies against Leishmania: IFAT titres $1 / 320$ to $1 / 5120$ and had symptoms common to both diseases: unusual tiredness, ocular lesions, weight loss. Twenty-four of these cultures contained 5-6 $\mathrm{L} 1 \mathrm{~mL}^{-1}$ live and active Dirofilaria, which appeared on the first day and stayed alive until over 30 days at $26 \pm 1{ }^{\circ} \mathrm{C}$. From the other two L1 positive cultures, 1 and $2 \mathrm{~L}_{1} \mathrm{~mL}^{-1}$ emerged on day 1 . In the culture containing 1 microfilariae $\mathrm{mL}^{-1}$, the microfilariae died on day 4 of the 


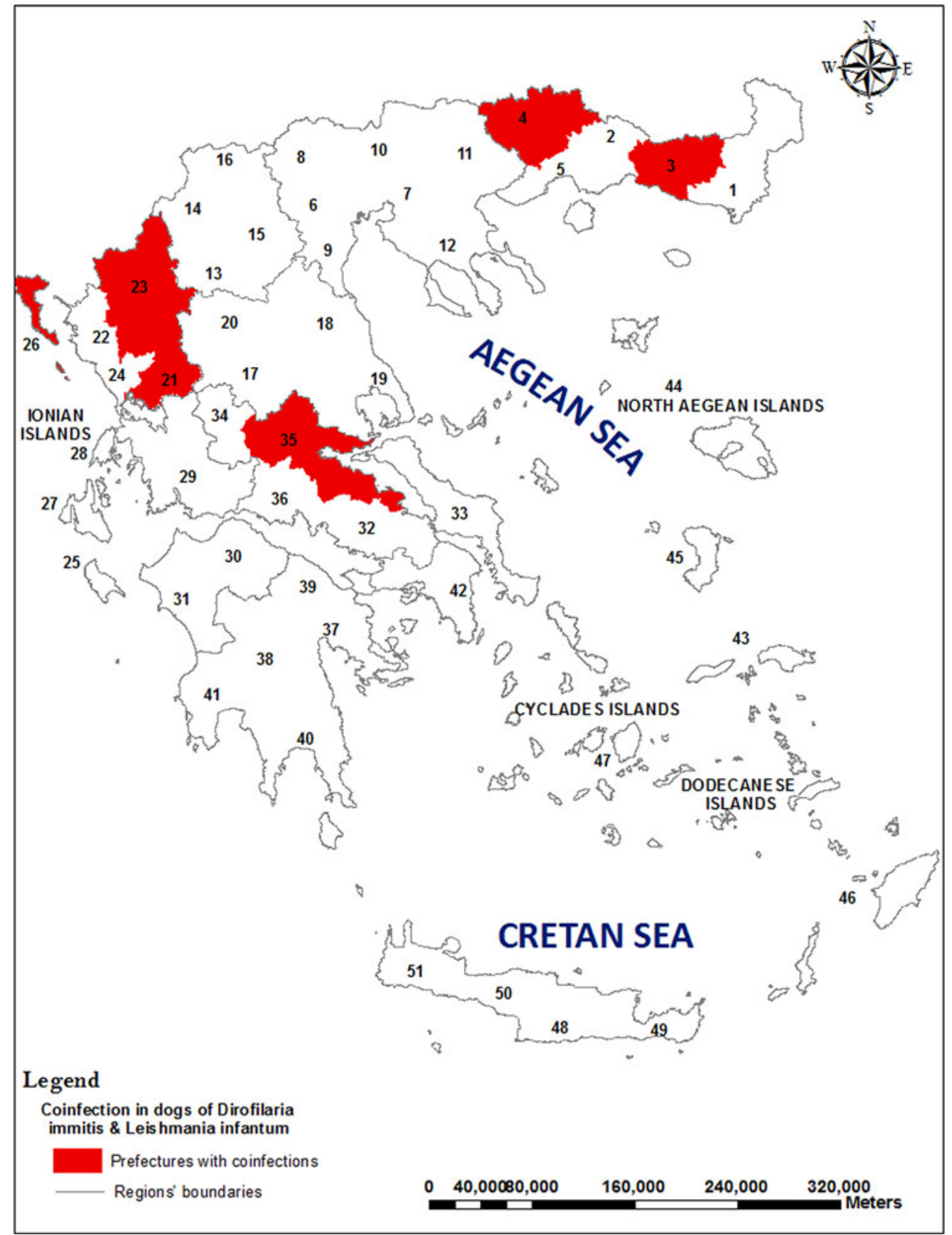

Fig. 1. Leishmania infantum and Dirofilaria immitis coinfections in dogs in Greece. L1 of D. immitis were isolated from clotted blood of dogs which were positive for L. infantum by serology and PCR from prefectures: Rodopi (indicated by number 3), Drama (4), Arta (21), Ioannina (23), Corfu (26), Fthiotida (35).

culture and on day 7, Leishmania promastigotes appeared, grew in numbers and propagated successfully. In the second culture containing $2 \mathrm{~L} 1 \mathrm{~mL}^{-1}$, a small number of Leishmania promastigotes emerged on day 8 , but disappeared after about 1 week, whilst the L1 survived for 30 days.

In the 165 cultures which yielded Leishmania promastigotes, the parasites propagated indefinitely in the RPMI medium at $26 \pm 2{ }^{\circ} \mathrm{C}$ until the culture was terminated after the second passage to be frozen in liquid nitrogen for future work.

The Dirofilaria and Leishmania coinfected dogs originated from five prefectures (nine dogs from Corfu, seven from Drama, five from Fthiotida, two from Arta, two from Ioannina, one from Rodopi) in which CanL is endemic (Fig. 1).

\section{DISCUSSION}

Leishmaniasis is recognized as a very important disease for public health in the whole of the Mediterranean Basin, including Greece where it is notifiable. It appears that it has spread geographically nearly in the whole of the country (Antoniou et al. 2009; Ntais et al. 2013) implying that competent sand fly vectors are present and active. The problem of dirofilariasis in Greece, on the other hand, is not considered very important for public 
health since a few sporadic human cases have being reported which, however, are increasing in number (Vakalis and Himonas, 1997). The presence of microfilaraemic dogs in an area, and possibly of other domestic and wild reservoirs, such as the red foxes (Karayiannis et al. 2015), allows the circulation of the parasite via the mosquito vectors, which explains the escalation of human cases and poses a thread for the future. The spread of both zoonoses is further favoured by the movement of people and pets and the spread of the vectors in space and time due to climatic changes, as well as human activities.

Measures for safeguarding unaffected areas from the introduction of these pathogens (through infected humans or animals) and their vectors must be undertaken. The introduction of Leishmania donovani MON-37 in Cyprus (Antoniou et al. 2008) and the arrival of the aggressive anthropophilic mosquito Aedes albopictus in Europe, which can play the role of the vector for $D$. immitis and could increase the risk of transmission of this parasite from animals to humans (Cancrini et al. 2003), is an indication of the enhanced movement of pathogens by globalization.

The culture method used is fast, simple and inexpensive and allows the isolation of both parasites from a big number of samples during an epidemiological study for monitoring risk areas. In this study, $12.94 \%$ of the dogs studied were positive for Leishmania and $2 \cdot 04 \%$ were positive for Leishmania and Dirofilaria. Coinfections were found in the prefectures of Corfu, Drama, Fthiotida, Arta, Ioannina and Rodopi (Fig. 1). Coinfected red foxes have also being reported in Fthiotida prefecture by Karayiannis et al. (2015).

Although filarial worms, including Dirofilaria, have being maintained in vitro (Silverman and Hansen, 1971), as far as we know, this is the first record of isolation of live Dirofilaria L1 from the blood of a host and their maintenance for at least 1 month in the RPMI medium at $26^{\circ} \mathrm{C}$. The method can also be used for the isolation of Leishmania promastigotes from biological samples (blood without anticoagulants, spleen tissue and lymph node), by placing a small segment of the sterile tissue in the RPMI culture medium. This method can be used to isolate L. infantum and L. tropica from biological samples without the need of a density gradient cell separation medium for the acquisition of lymphocytes to be used for the culture.

It is interesting to note that only in two out of the 26 IFAT and PCR positive for Leishmania samples in which Dirofilaria L1 emerged, Leishmania promastigotes appeared. In one of these two cases, promastigotes proliferated successfully after the three L1 (in the $3 \mathrm{~mL}$ culture medium) died, but the weak Leishmania culture which appeared on day 8 of the second culture died out after 1 week in the presence of seven L1 in the culture medium. It would be interesting to investigate whether the presence of microfilariae do not favour promastigote proliferation and survival for long in the culture medium and possibly in the host as well. The microfilariae may feed on the promastigotes or produce metabolic substances that do not allow the protozoan parasite to exist. This could allow the development of potential new substances in combating leishmaniasis in dogs and humans.

\section{ACKNOWLEDGEMENTS}

The authors wish to thank all veterinarians who helped in obtaining the dog biological samples and data.

\section{FINANCIAL SUPPORT}

We wish to thank EDENext for funding the work (EU grant FP7-261504 to M. A.). The manuscript was catalogued by the EDENext Steering Committee as EDENext388 (http://www.edenext.eu). The contents of this publication are the sole responsibility of the authors and don't necessarily reflect the views of the European Commission.

\section{REFERENCES}

Alvar, J., Velez, I. D., Bern, C., Herrero, M., Desjeux, P., Cano, J., Jannin, J. and den Boer, M. (2012). Leishmaniasis worldwide and global estimates of its incidence. PLoS ONE 7, e35671.

Antoniou, M., Haralambous, C., Mazeris, A., Pratlong, F., Dedet, J. P. and Soteriadou, K. (2008). Leishmania donovani leishmaniasis in Cyprus. The Lancet Infectious Diseases 8, 6-7.

Antoniou, M., Messaritakis, I., Christodoulou, V., Ascoksilaki, I., Kanavakis, N., Sutton, A. J., Carson, C. and Courtenay, O. (2009). Increasing incidence of zoonotic visceral leishmaniasis on Crete, Greece. Emerging Infectious Diseases 15, 932-934.

Aresu, L., Valenza, F., Ferroglio, E., Pregel, P., Uslenghi, F., Tarducci, A. and Zanatta, R. (2007). Membranoproliferative glomerulonephritis type III in a simultaneous infection of Leishmania infantum and Dirofilaria immitis in a dog. Fournal of Veterinary Diagnostic Investigation 19, 569-572.

Cancrini, G., Frangipane di Regalbono, A., Ricci, I., Tessarin, C., Gabrielli, S. and Pietrobelli, M. (2003). Aedes albopictus is a natural vector for Dirofilaria immitis in Italy. Veterinary Parasitology 118, 195-202. Christodoulou, V., Antoniou, M., Ntais, P., Messaritakis, I., Ivovic, V., Dedet, J. P., Pratlong, F., Dvorak, V. and Tselentis, Y. (2012). Re-emergence of visceral and cutaneous leishmaniasis in the Greek Island of Crete. Vector-Borne and Zoonotic Diseases 12, 214-222.

Dujardin, J. C. (2006). Risk factors in the spread of leishmaniases: towards integrated monitoring? Trends in Parasitology 22, 4-6.

Ferroglio, E., Trisciuoglio, A., Gastaldo, S., Mignone, W. and Delle Piane, M. (2002). Comparison of ELISA, IFAT, and Western blot for the serological diagnosis of Leishmania infantum infection in $\mathrm{dog}$. Parassitologia 44 (Suppl. 1), 64.

Genchi, C., Kramer, L. H. and Rivasi, F. (2011). Dirofilarial infections in Europe. Vector Borne and Zoonotic Diseases 10, 1307-1317.

Howard, M. K., Pharoah, M. M., Ashall, F. and Miles, M. A. (1991). Human urine stimulates growth of Leishmania in vitro. Transactions of the Royal Society of Tropical Medicine and Hygiene 85, 477-479.

Karayiannis, S., Ntais, P., Messaritakis, I., Tsirigotakis, N., Dokianakis, E. and Antoniou, M. (2015). Detection of Leishmania infantum in red foxes (Vulpes vulpes) in central Greece. Parasitology 24, 1-5.

Magnis, J., Lorentz, S., Guardone, L., Grimm, F., Magi, M., Naucke, T. J. and Deplazes, P. (2013). Morphometric analyses of canine blood microfilariae isolated by the Knott's test enables Dirofilaria and Acanthocheilonema (syn. Dipetalonema) immitis and D. repensspeciesspecific genus-specific diagnosis. Parasites and Vectors 6, 48.

Maia, C., Coimbra, M., Ramos, C., Cristóvão, J. M., Cardoso, L. and Campino, L. (2015). Serological investigation of Leishmania infantum, 
Dirofilaria immitis and Angiostrongylus vasorum in dogs from southern Portugal. Parasites and Vectors 8, 152.

Maroli, M., Rossi, L., Baldelli, R., Capelli, G., Ferroglio, E., Genchi, C., Gramiccia, M., Mortarino, M., Pietrobelli, M. and Gradoni, L. (2008). The northward spread of leishmaniasis in Italy: evidence from retrospective and ongoing studies on the canine reservoir and phlebotomine vectors. Tropical Medicine \& International Health 13, 256-264. McCall, J. W., Genchi, C., Kramer, L. H., Guerrero, J. and Venco, L. (2008). Heartworm disease in animals and humans. Advances in Parasitology 66, 193-285.

Minodier, P., Piarroux, R., Gambarelli, F., Joblet, C. and Dumon, H. (1997). Rapid identification of causative species in patients with Old World leishmaniasis. Fournal of Clinical Microbiology 35, 2551-2555.

Ntais, P., Sifaki-Pistola, D., Christodoulou, V., Messaritakis, I., Pratlong, F., Poupalos, G. and Antoniou, M. (2013). Leishmaniases in Greece. American fournal of Tropical Medicine and Hygiene 89, 906-915.

Orihel, T. A. and Eberhard, M. L. (1998). Zoonotic filariasis. Clinical Microbiology Reviews 11, 366-381.

Otto, G. F. and Jachowski, L. A. (1981). Mosquitoes and canine heartworm disease. In Proceedings of the Heartworm Symposium 80 (ed. Otto, G. F.), pp. 17-32. Veterinary Medicine Publishing Co, Edwardsville, Kansas.

Pampiglione, S. and Rivasi, F. (2000). Human dirofilariasis due to Dirofilaria (Nochtiella) repens: an update of world literature from 1995 to 2000. Parassitologia 42, 235-254.
Polizopoulou, Z. S., Koutinas, A. F., Saridomichelakis, M. N., Patsikas, M. N., Leontidis, L. S., Roubies, N. A. and Desiris, A. K. (2000). Clinical and laboratory observations in 91 dogs infected with Dirofilaria immitis in northern Greece. Veterinary Record 146, 466-469. Schönian, G., Nasereddin, A., Dinse, N., Schweynoch, C. Schallig, H. D., Presber, W. and Jaffe, C. L. (2003). PCR diagnosis and characterization of Leishmania in local and imported clinical samples. Diagnostic Microbiology and Infectious Disease 47, 349-358.

Silverman, P. H. and Hansen, E. L. (1971). In vitro cultivation procedures for parasitic helminths: recent advances. Advances in Parasitology 9, 227-258. Sinanis, T. N., Koutinas, C. K., Diakou, A. and Papadopoulou, P. (2012). Canine heartworm disease (dirofilariosis): pathogenesis and diagnosis of a multidimensional disease. Fournal of the Hellenic Veterinary Medical Society 63, 291-300.

Tanczos, B., Balogh, N., Kiraly, L., Biksi, I., Szeredi, L., Gyurkovsky, M., Scalone, A., Fiorentino, E., Gramiccia, M. and Farkas, R. (2012). First record of autochthonous canine leishmaniasis in Hungary. Vector Borne and Zoonotic Diseases 12, 588-594.

Vakalis, N. C. and Himonas, C. A. (1997). Human and canine dirofilariasis in Greece. Parassitologia 39, 389-391.

World Health Organization (1991). Basic laboratory methods. In Medical Parasitology. WHO, Geneva.

World Health Organization (1997). Inf. Circ.-WHO Mediterranean Zoonoses Control Cent 44, 58-61. http://apps.who.int/iris/bitstream/10665/ 40793/1/9241544104_(part1).pdf. 